OPEN ACCESS

Edited by:

Gary W. Mack,

Brigham Young University,

United States

Reviewed by:

Zachary Schlader,

University at Buffalo, United States

Kei Nagashima,

Waseda University, Japan

Keiii Hayashi,

University of Shizuoka, Japan

*Correspondence:

Ahmad Munir Che Muhamed

ahmadmunir@usm.my

Specialty section:

This article was submitted to

Exercise Physiology,

a section of the journal

Frontiers in Physiology

Received: 03 November 2018

Accepted: 11 April 2019

Published: 07 May 2019

Citation:

Che Muhamed AM, Yusof HA,

Stannard SR, Mündel $T$ and Thompson MW (2019) The Efficacy

of Ingesting Water on

Thermoregulatory Responses and Running Performance in a

Warm-Humid Condition.

Front. Physiol. 10:507.

doi: 10.3389/fphys.2019.00507

\section{The Efficacy of Ingesting Water on Thermoregulatory Responses and Running Performance in a Warm-Humid Condition}

\author{
Ahmad Munir Che Muhamed ${ }^{1 *}$, Hazwani Ahmad Yusof', Stephen R. Stannard ${ }^{2}$, \\ Toby Mündel ${ }^{2}$ and Martin William Thompson ${ }^{3}$ \\ 'Lifestyle Science Cluster, Advanced Medical and Dental Institute, Universiti Sains Malaysia, Penang, Malaysia, ${ }^{2}$ School \\ of Sport, Exercise and Nutrition, Massey University, Palmerston North, New Zealand, ${ }^{3}$ Discipline of Exercise and Sport \\ Science, Faculty of Health Science, The University of Sydney, Sydney, NSW, Australia
}

The understanding that fluid ingestion attenuates thermoregulatory and circulatory stress during exercise in the heat was based on studies conducted in relatively dry $(\sim 50 \% \mathrm{RH})$ environments. It remains undetermined whether similar effects occur during exercise in a warm and more humid environment, where evaporative capacity is reduced. Nine well-trained, unacclimatised male runners were randomly assigned to perform four experimental trials where they ran for 60 min at an intensity of $70 \%$ $\mathrm{VO}_{2}$ max followed by an incremental exercise test until volitional exhaustion. The four trials consisted of non-fluid ingestion (NF) and fluid ingestion (FI) in a warm-dry (WD) and warm-humid condition $(\mathrm{WH})$. Time to exhaustion (TTE), body temperature $\left(T_{b}\right)$, whole body sweat rate, partitional calorimetry measures, heart rate and plasma volume were recorded during exercise. There was no significant difference in $T_{b}$ following 60 min of exercise in $\mathrm{Fl}$ and $\mathrm{NF}$ trial within both WD $\left(37.3^{\circ} \mathrm{C} \pm 0.4\right.$ vs. $\left.37.4^{\circ} \mathrm{C} \pm 0.3 ; p>0.05\right)$ and $\mathrm{WH}$ conditions $\left(38.0^{\circ} \mathrm{C} \pm 0.4 \mathrm{vs} .38 .1^{\circ} \mathrm{C} \pm 0.4 ; p>0.05\right)$. The TTE was similar between $\mathrm{Fl}$ and $\mathrm{NF}$ trials in both $\mathrm{WH}$ and $\mathrm{WD}$, whereas exercise capacity was significantly shorter in WH than WD $(9.1 \pm 2.8 \mathrm{~min}$ vs. $12.7 \pm 2.4 \mathrm{~min}$, respectively; $p=0.01)$. Fluid ingestion failed to provide any ergogenic benefit in attenuating thermoregulatory and circulatory stress during exercise in the WH and WD conditions. Consequently, exercise performance was not enhanced with fluid ingestion in the warm-humid condition, although the humid environment detrimentally affected exercise endurance.

Keywords: fluid ingestion, thermoregulation, circulation, relative humidity, running exercise

\section{INTRODUCTION}

Recent evidence demonstrates a reduced physical capacity of trained individuals during exercise in the heat as relative humidity (RH) increases (Maughan et al., 2012; Moyen et al., 2014; Che Muhamed et al., 2016). This observation is attributed to a greater thermoregulatory and circulatory strain (Moyen et al., 2014; Che Muhamed et al., 2016), as the evaporative capacity of the environment $\left(\mathrm{E}_{\max }\right)$ reduces, leading to a decline in sweating efficiency and increased area of 
skin wettedness and the subsequent rise in core temperature (Candas et al., 1979; Frye and Kamon, 1983; Alber-Wallerström and Holmér, 1985).

Numerous recommendations encourage athletes to ingest sufficient fluid in order to prevent a loss of $>2 \%$ of their body weight during exercise (Sawka et al., 2007, 2015). This recommendation was based on laboratory studies demonstrating that a body mass loss of more than $2 \%$ impairs aerobic exercise performance in a thermally stressful environment (Montain and Coyle, 1992; Sawka et al., 2015). On the other hand, numerous publications have refuted the claim that aerobic exercise performance is degraded with increased ( $>2 \%$ ) dehydration (Noakes, 2007; Goulet, 2011, 2013; Wall et al., 2013). A metaanalysis by Goulet (2011) had highlighted that exercise-induced dehydration by up to $4 \%$ of body weight loss does not alter exercise performance. Instead, drinking according to the dictate of thirst will potentially improve exercise performance (Noakes, 2007). While the debate on the efficacy of fluid ingestion in minimizing the decline of aerobic exercise performance in ambient heat continues, the value of fluid ingestion during humid conditions is not well understood.

Early reports of an attenuation in hyperthermia with fluid ingestion are based on studies conducted in a hot and relatively dry $(\sim 50 \% \mathrm{RH})$ environment with the large $\mathrm{E}_{\max }$ allowing for efficient sweat evaporation (Montain and Coyle, 1992; Below et al., 1995). By contrast, in a humid environment with a lower $E_{\max }$, sweat will drip off the skin instead of evaporating (Candas et al., 1979; Frye and Kamon, 1983; AlberWallerström and Holmér, 1985) and so "ineffective" fluid loss through sweating is much greater. Therefore, the proportion that fluid ingestion contributes to thermoregulation via increasing sweating rate would also be less. These observations on the sweating response and sweating efficiency might question the efficacy of fluid ingestion during prolonged exercise in a warm-humid condition. To date, Kay and Marino (2003) have reported that self-paced exercise performance in a hot-humid environment was not improved with fluid ingestion. In addition, Lee et al. (2010), had reported that fluid ingestion did not alter thermoregulatory responses during field-based experiments among military personnel. Therefore, the purpose of this study was to investigate the efficacy of ingesting plain water in attenuating the thermoregulatory and circulatory stress during prolonged moderate-high intensity exercise under a warmdry and warm-humid environment and its consequences on running performance.

\section{MATERIALS AND METHODS}

\section{Participants}

Nine well-trained, unacclimatised male runners who regularly participated in middle and long distance running events volunteered for this study. The subjects' characteristics were (mean $\pm \mathrm{SD}$ ): age, $32 \pm 4$ years; height, $183 \pm 6 \mathrm{~cm}$; weight, $72 \pm 4 \mathrm{~kg}$; body surface area, $1.9 \pm 0.1 \mathrm{~m}^{2}$; percent body fat, $11 \pm 6 \% ; \mathrm{VO}_{2} \max , 62 \pm 5 \mathrm{ml} \mathrm{kg}{ }^{-1} \mathrm{~min}^{-1}$. Participants were briefed on the experimental protocol and testing procedures before providing written informed consent to participate. The University of Sydney Human Ethics Committee approved the experimental protocol for this study (Ref. No: 99/05/46), which conformed to the current Declaration of Helsinki guidelines. All experiments were conducted in a purpose-built environmental chamber during the autumn and winter season in Sydney, Australia. The average outdoor temperature during the experimental trials was 20 to $22^{\circ} \mathrm{C}$ in autumn and 7 to $10^{\circ} \mathrm{C}$ during winter. None of the participants were accustomed to exercise in the conditions simulated in this study.

\section{Preliminary Testing and Familiarization}

Each participant visited the environmental chamber and was familiarized with the exercise protocol, equipment, and measurement procedures used in the study. This was followed by a preliminary testing session during which anthropometric measures, including body height, weight, composition, and surface area were determined. Body surface area was calculated using the method of DuBois and DuBois (1916). Percent body fat was measured using the hydrodensitometry underwater weighing technique described by Siri (1961).

Each participant then performed a running economy test followed by a maximal exercise test. The running economy test required each subject to run at four submaximal velocities of 10 , 12,14 , and $16 \mathrm{~km} \mathrm{~h}^{-1}$, respectively, for $4 \mathrm{~min}$ per stage. On completion of the submaximal running, participants engaged in an active recovery, where they walked for $5 \mathrm{~min}$ at a speed of $5 \mathrm{~km}$ $\mathrm{h}^{-1}$. This was followed by a graded exercise test to determine maximal oxygen uptake $\left(\mathrm{VO}_{2} \max \right)$, where the participant ran at a fixed speed of $12 \mathrm{~km} \mathrm{~h}^{-1}$ with the treadmill gradient elevated by $2 \%$ every 2 min until volitional fatigue was attained. Oxygen consumption and heart rate were taken throughout the exercise duration. Post-exercise, a linear regression line was plotted between submaximal steady state oxygen consumption and treadmill velocity to determine the participant's running speed that elicited an intensity of $70 \% \mathrm{VO}_{2} \mathrm{max}$, which was used for the individual's experimental trials for each subsequent testing session. The preliminary and familiarization sessions were conducted in a thermoneutral environment $\left(20^{\circ} \mathrm{C}, 40 \% \mathrm{RH}\right)$.

Prior to reporting to the laboratory for testing, each participant was reminded to refrain from heavy exercise and alcohol consumption the day before testing and to avoid caffeine consumption for $12 \mathrm{~h}$ before the test. They were also told to maintain a similar training routine throughout the duration of the study. Participants were asked to keep a $24 \mathrm{~h}$ food diary before testing and replicate their diet before subsequent visits to the laboratory. To ensure that participants were euhydrated at the onset of exercise, they were asked to ingest $6 \mathrm{ml}$ of water per $\mathrm{kg}$ lean body mass at $2 \mathrm{~h}$ intervals (excluding when asleep) during the day before the test as well as the morning of testing.

\section{Experimental Protocol}

Each participant ran for $60 \mathrm{~min}$ at a speed eliciting an intensity of $70 \% \mathrm{VO}_{2} \max$. Immediately thereafter, participants continued running at the same velocity while the treadmill gradient was elevated by $2 \%$ every 2 min until volitional exhaustion, defined as the point at which participants could no longer maintain the 
pace of the treadmill, upon which the test was terminated. During the 60 min submaximal exercise, the treadmill was briefly stopped ( $1 \mathrm{~min}$ ) at 30 and $60 \mathrm{~min}$ to allow time for the determination of the participant's body mass. The air speed set for each trial was matched to the individual running speed and thus simulated the effect of air resistance on a calm day outdoors. Each test session was separated by a week apart to minimize acclimation effects as well as to provide adequate recovery.

The experiment started with participants being randomly assigned to perform the running exercise without ingesting water (non-fluid ingestion trial; NF) across two environmental conditions of warm-dry (WD: $30^{\circ} \mathrm{C}$ and $24 \% \mathrm{RH}$ ) and warmhumid (WH: $30^{\circ} \mathrm{C}$ and $\left.71 \% \mathrm{RH}\right)$. Following the $\mathrm{NF}$ trial, participants were randomly assigned to the same exercise regimen while being allowed to ingest water (fluid ingestion trial; FI) across the WD and WH conditions. The NF trials enabled the determination of each individual subject's sweat rate within the specific environmental condition for the calculation of the fluid volume needed to replace $80 \%$ of sweat loss during the FI trial.

At least 1 week elapsed between each of the subject's four scheduled visits to the laboratory, in order to minimize any residual effects from the previous visit. To avoid any psychological apprehension, subjects were not informed of the environmental condition on the day of the test.

\section{Fluid Ingestion Protocol}

In the FI trial, water amounting to $80 \%$ of total sweat loss from the NF trial was equally divided into five aliquots and provided to participants during submaximal exercise, starting at $10 \mathrm{~min}$ and followed at every $10 \mathrm{~min}$ interval with the final ingestion at $50 \mathrm{~min}$. The volume of fluid ingestion in this study which was based on $80 \%$ of total sweat loss was about $1 \mathrm{~L}$ (ranged between 1.12 and $0.96 \mathrm{~L}$ ). This amount of fluid ingestion was consistent with the water requirements for an hour of running exercise at a similar metabolic rate in a hot and humid condition as previously described by Sawka and Pandolf (1990). The temperature of the water ingested at each time interval was maintained at $15^{\circ} \mathrm{C}$.

\section{Measurements}

As an index of core temperature, rectal temperature $\left(\mathrm{T}_{\mathrm{re}}\right)$ was measured by a thermistor probe (YSI 400 series; Mallinckrodt Medical, St. Louis, MO, United States) inserted $12 \mathrm{~cm}$ beyond the anal sphincter and data were recorded on a portable data logger (T-logger; The University of Sydney, Sydney, NSW, Australia). Skin temperature was measured at four different sites (left shoulder, left chest, right mid-thigh, and right midshin) using thermistor probes (YSI 409 Series). Rectal and skin temperatures were sampled at $1 \mathrm{~min}$ intervals. Weighted mean skin temperature $\left(\overline{\mathrm{T}}_{\mathrm{sk}}\right)$ was calculated from the four sites (Ramanathan, 1964). Mean body temperature $\left(\mathrm{T}_{\mathrm{b}}\right)$ was calculated using the equation developed by Colin et al. (1971) as $\mathrm{T}_{\mathrm{b}}=0.79$ $\left(\overline{\mathrm{T}}_{\mathrm{re}}\right)+0.21\left(\overline{\mathrm{T}}_{\mathrm{sk}}\right)$. Both rectal and skin thermistor probes used in the study were calibrated before and after the study in a water bath with temperature ranging from 15 to $50^{\circ} \mathrm{C}$; calculated accuracies were \pm 0.05 and \pm 0.01 , respectively.

Expired respiratory gas was obtained using the Douglas bag method at rest and at every 10 min intervals of the submaximal exercise and analyzed for fractions of $\mathrm{O}_{2}$ and $\mathrm{CO}_{2}$ concentration using gas sensors and analyzers $\left(\mathrm{O}_{2}\right.$ analyzer, Ametek S-3A/I and $\mathrm{CO}_{2}$ analyzers, Ametek CD-3A, Applied Electrochemistry Ametek Inc., Thermox Instruments Division). The gas analyzers were calibrated with known calibration gasses prior to each testing session.

Cardiac output during the steady state exercise phase was determined at 10,30 and $60 \mathrm{~min}$ by the $\mathrm{CO}_{2}$ rebreathing method (Collier, 1956). Stroke volume was then calculated using the Fick equation. Heart rate was continuously monitored telemetrically via a Polar transmitter-receiver (Polar Vantage XL, Polar Electro, Kempele, Finland) and recorded at rest, during submaximal exercise at $5 \mathrm{~min}$ intervals and at volitional exhaustion.

Whole body sweating rate (WBSR) during steady state exercise was calculated as the difference in pre and post $60 \mathrm{~min}$ exercise nude body weight with correction for respiratory moisture loss (Mitchell et al., 1972) and incorporating volume of fluid consumed. Participant's perception of the difficulty of the exercise effort was recorded based on the 6-20 point RPE scale (Borg, 1982) and was recorded at 10 min intervals.

A partitional calorimetry software program developed by Atkins and Thompson (2000) was used to estimate the magnitude and avenue of body heat gain and heat loss during each of the prolonged exercise bouts. Partitional calorimetry calculations were based on measures taken within the last $30 \mathrm{~min}$ of submaximal exercise of each trial. Evaporative heat loss in $\mathrm{W}$ $\mathrm{m}^{-2}$ was estimated from the classic body heat balance equation of $\mathrm{E}=\mathrm{M}-\mathrm{W}$ (Eres + Cres) $-\mathrm{R}-\mathrm{C}-\mathrm{S}$, where $\mathrm{M}$ is metabolic heat production, $\mathrm{W}$ is external work, Eres + Cres is heat transfer via the respiratory tract, $\mathrm{R}$ is radiative heat loss, $\mathrm{C}$ is convective heat loss, and $\mathrm{S}$ is body heat storage. $\mathrm{S}$ was estimated using the following equation: $\left[3474 \times \mathrm{wt} \times\left(\mathrm{T}_{\mathrm{b}}\right.\right.$ final $\mathrm{T}_{\mathrm{b}}$ initial $\left.) \times \mathrm{t}^{-1}\right] \times \mathrm{A}_{D}{ }^{-1}\left(\mathrm{~W} \mathrm{~m}^{-2}\right)$, where 3474 is the average specific heat of body tissue $\left(\mathrm{J} \mathrm{kg}^{-1}{ }^{\circ} \mathrm{C}^{-1}\right)$, wt is body mass $(\mathrm{kg}), \mathrm{T}_{\mathrm{b}}$ is mean body temperature $\left({ }^{\circ} \mathrm{C}\right), \mathrm{t}$ is exercise time $(\mathrm{sec})$, and $A_{D}$ is body surface area $\left(\mathrm{m}^{2}\right)$. Estimation of tissue heat conductance (K) was derived from the method of Davies (1979)

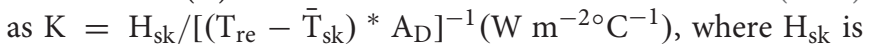
the heat dissipated from the skin $(\mathrm{E}+\mathrm{R}+\mathrm{C})$, Tre is rectal temperature, $\mathrm{T}_{\text {sk }}$ is mean skin temperature, and $\mathrm{A}_{D}$ is the DuBois body surface area in $\mathrm{m}^{-2}$. Convective and radiative heat loss in $\mathrm{W}$ $\mathrm{m}^{-2}$ were estimated using the equations found in Fanger (1970) and McIntyre (1980), respectively.

\section{Blood Sampling and Analysis}

Venous blood was sampled at rest and 10, 30, and $60 \mathrm{~min}$ of submaximal exercise and were immediately stored for analysis of hematocrit and hemoglobin (CIBA-Corning 800 Series). Analysis for hematocrit percentage and hemoglobin concentration were analyzed in triplicate and used to estimate percentage changes in resting plasma volume based on the method of Dill and Costill (1974).

\section{Statistical Analysis}

A two way (humidity $\mathrm{x}$ fluid treatment) repeated measures analysis of variance (ANOVA) was used to compare the differences between the means of the data measured in this study. 

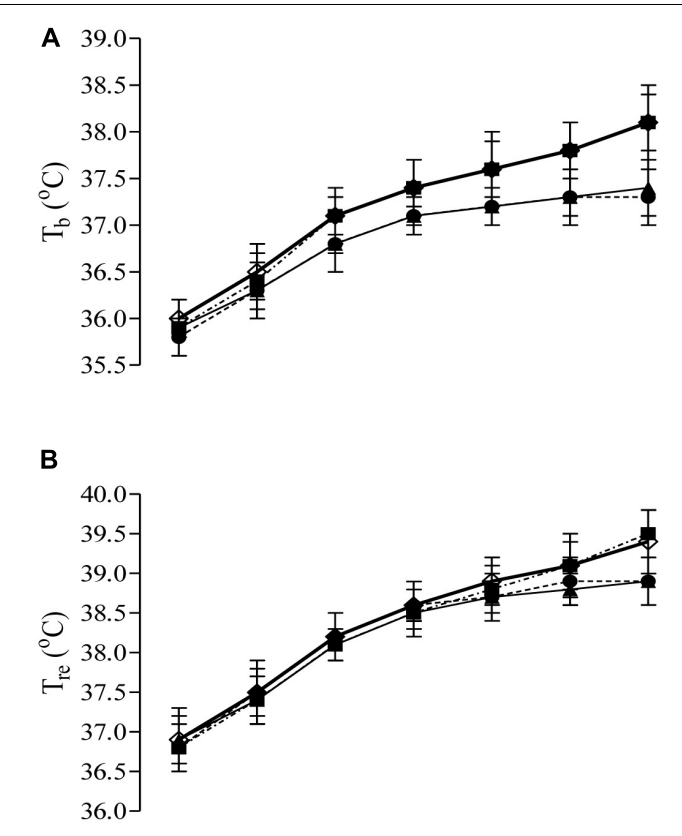

C

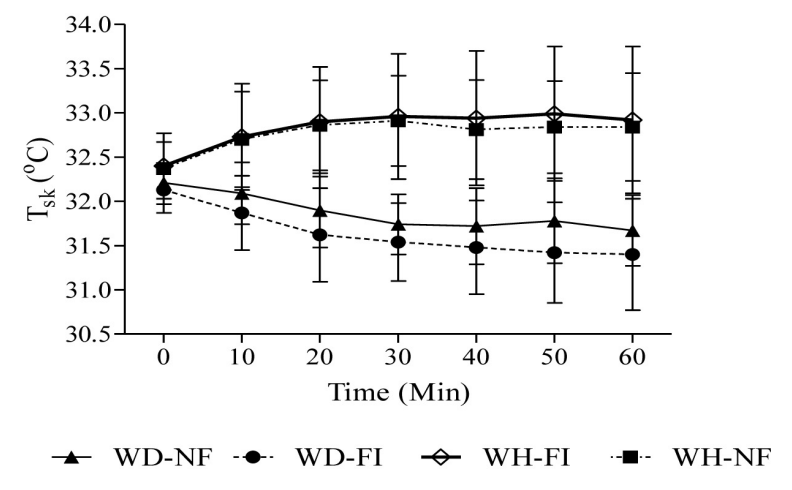

FIGURE 1 | Mean body temperature (A), rectal temperature (B), and weighted mean skin temperature $\mathbf{( C )}$ responses during the steady state exercise in the fluid ingestion (FI) and non-fluid ingestion trial (NF) in a warm-dry (WD) and warm-humid (WH) condition.

If a primary significant difference was observed, the post hoc Tukey's paired $t$-test with a Bonferroni correction for multiple comparisons was used to detect where the differences occurred. A Huynh-Feldt correction was applied to adjust the degrees of freedom when the test of sphericity was significant. Statistics were analyzed using SPSS statistical software (V22.0, Chicago, IL, United States), with statistical significance accepted at an $\infty$ level of 0.05 . Data reported are presented as means $\pm \mathrm{SD}$.

\section{RESULTS}

\section{Steady-State Exercise}

Responses of $\mathrm{T}_{\mathrm{re}}, \mathrm{T}_{\mathrm{sk}}$ and $\mathrm{T}_{\mathrm{b}}$ can be seen in Figure 1. ANOVA revealed no significant main effect of fluid ingestion on $\mathrm{T}_{\mathrm{re}}, \mathrm{T}_{\text {sk }}$ or $\mathrm{T}_{\mathrm{b}}$ (all $p>0.2$ ), however, a main effect of humidity was observed for all (all $p<0.02$ ) during the steady state exercise. At the end of steady state exercise, a significantly higher $\mathrm{T}_{\mathrm{b}}$ was recorded in WH as compared with WD for both $\mathrm{FI}\left(38.0^{\circ} \mathrm{C} \pm 0.4\right.$ vs. $\left.37.3^{\circ} \mathrm{C} \pm 0.3 ; p<0.01\right)$ and NF trials $\left(38.1^{\circ} \mathrm{C} \pm 0.4\right.$ vs. $\left.37.4^{\circ} \mathrm{C} \pm 0.3 ; p<0.01\right)$.

WBSR differed between fluid trials as a function of ambient humidity (fluid ingestion $\mathrm{x}$ ambient humidity: $p<0.01$ ), such that NF in WD resulted in similar WBSR during exercise when compared with FI (24.5 $\mathrm{g} \mathrm{min}^{-1} \pm 2.3$ vs. $24.3 \mathrm{~g} \mathrm{~min}^{-1} \pm 2.3$, respectively; $p=0.6$ ), whilst in WH WBSR was significantly higher in FI compared to NF $\left(26.1 \mathrm{~g} \mathrm{~min}^{-1} \pm 4.8\right.$ vs. $22.7 \mathrm{~g}$ $\min ^{-1} \pm 3.4$, respectively; $\left.p=0.01\right)$. Percent body weight deficit was significantly larger in the NF as compared with the FI trial $(p<0.01)$, within both WD $(2.0 \pm 0.2$ vs. $0.4 \pm 0.1$, respectively) and WH environments ( $1.8 \pm 0.3$ vs. $0.6 \pm 0.2)$, although ambient humidity had no effect $(p=0.6)$. The change in PV (measured between 10th and 60th minutes) was similar between FI and NF trials $(2.1 \pm 1.8 \%$ vs. $4.2 \pm 3.0 \%$, respectively; $p=0.1)$ although the change in PV differed as a consequence of ambient humidity $(2.0 \pm 1.4 \%$ vs. $4.2 \pm 2.9 \%$, respectively; $p=0.04)$.

There was no significant effect of fluid ingestion on RPE during exercise $(p=0.4)$, however, ambient humidity significantly affected RPE $(p<0.01)$. Participants reported exercise as being harder to perform in the WH environment as compared with WD, respectively, for both NF (16 \pm 2 vs. $14 \pm 2$, respectively) and FI trials ( $15 \pm 2$ vs. $13 \pm 2$, respectively).

ANOVA revealed a significantly higher $S$ during NF than FI $\left(6 \pm 7 \mathrm{~W} \mathrm{~m}^{-2} ; p=0.03\right)$ and $\mathrm{WH}$ than WD $\left(22 \pm 9 \mathrm{~W} \mathrm{~m}^{-2}\right.$; $p<0.01)$. $\mathrm{M}$ was significantly higher during $\mathrm{WH}$ than WD $\left(23 \pm 27 \mathrm{~W} \mathrm{~m} \mathrm{~m}^{-2} ; p=0.03\right)$ with no effect of fluid ingestion $(p=0.3)$, whilst $\mathrm{E}$ was significantly higher during FI than NF $\left(14 \pm 15 \mathrm{~W} \mathrm{~m}^{-2} ; p=0.03\right)$ with no effect of ambient humidity $(p=0.2) . \mathrm{K}$ and $\mathrm{C}+\mathrm{R}$ were both higher during $\mathrm{WH}$ than WD $\left(22 \pm 12\right.$ and $25 \pm 8 \mathrm{~W} \mathrm{~m}^{-2}$, respectively; both $\left.p<0.01\right)$ with no effect of fluid ingestion (both $p>0.2$ ).

The circulatory responses can be seen in Figure 2. ANOVA revealed a significant effect of fluid ingested $(p=0.01)$ and ambient humidity $(p<0.01)$ on heart rate, such that on average FI reduced HR by $2 \pm 2$ beats $\min ^{-1}$ whilst on average the WH environment increased HR by $7 \pm 5$ beats $\min ^{-1}$. Stroke volume was lower during WH than WD by $7 \pm 5 \mathrm{ml}(p<0.01)$ with no effect of fluid ingested $(p=0.7)$. No effects of fluid ingestion or ambient humidity were observed on cardiac output (both $p>0.5$ ).

\section{Graded Exercise to Exhaustion}

Fluid ingestion did not have any effect on the time to exhaustion within both conditions when compared to the no fluid ingestion trial as presented in Figure 3. The exercise capacity during the graded exercise test within both NF and FI trials was significantly shorter in WH than WD $(9.1 \pm 2.8 \mathrm{~min}$ vs. $12.7 \pm 2.4 \mathrm{~min}$, respectively; $p=0.01$ ). At exhaustion, $\mathrm{T}_{\text {re }}$ was not significantly different between the FI and the $\mathrm{NF}$ or WD and WH trials (global mean $39.3 \pm 0.5^{\circ} \mathrm{C}$; all $p>0.2$ ). Similarly, $\mathrm{T}_{\mathrm{sk}}$ (global mean $32.1 \pm 1.2^{\circ} \mathrm{C}$; all $p>0.1$ ) and heart rate (global mean $182 \pm 1$ beats 


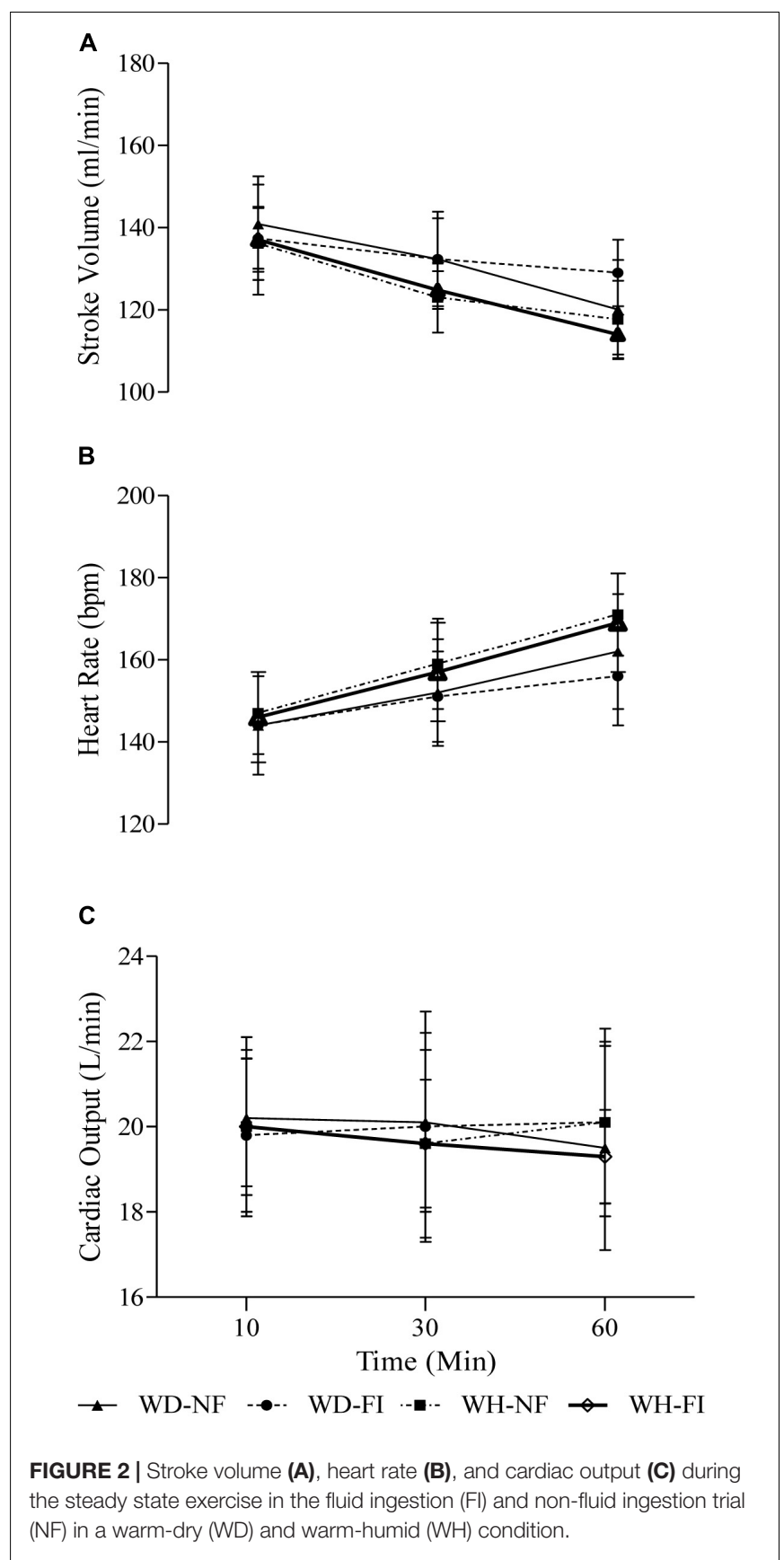

$\min ^{-1}$; all $p>0.3$ ) were also not significantly different between any condition.

\section{DISCUSSION}

To our knowledge, this is the first study to directly examine the efficacy of fluid ingestion in attenuating thermoregulatory strain during prolonged exercise in two different environments: warm-dry (WD) and warm-humid (WH). This study was designed to simulate real outdoor running where runners would experience a high level of air velocity during their

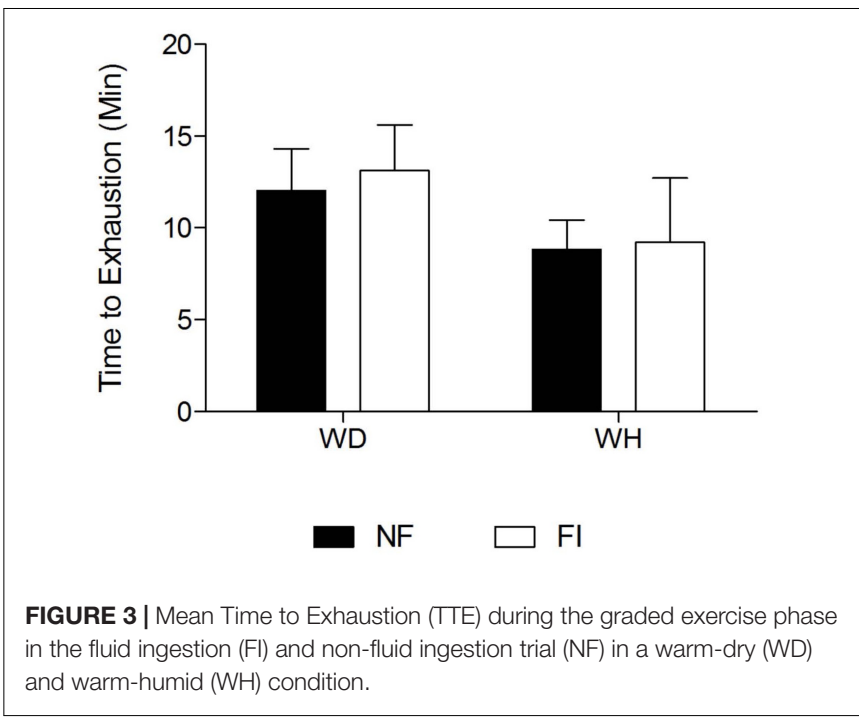

intense running exercise, thus promoting convective cooling. The main finding of this study was that fluid ingestion during exercise in warm-humid and warm-dry conditions did not attenuate hyperthermia during exercise. Consequently, the time to exhaustion in the subsequent graded exercise session was not improved during the FI trial. Under this current scenario, fluid ingestion was seen not to provide any beneficial effect on thermoregulatory and circulatory responses and consequently on exercise performance.

This study had also postulated that the level of percent body mass deficit did not influence the magnitude of physiological strain during submaximal exercise and running performance during the graded exercise test. In the present experiment, the percent body mass deficit during the no fluid (NF) trial, in the WD and WH condition was 2 and $1.8 \%$, respectively.

Therefore, the current study provides evidence that thermoregulatory responses during the submaximal exercise could have been influenced by the level of $\mathrm{RH}$ as previously reported (Maughan et al., 2012; Moyen et al., 2014; Che Muhamed et al., 2016) and not associated with the level of dehydration as advocated in numerous publications (Noakes, 2007; Goulet, 2011, 2013; Wall et al., 2013). This current observation is contrary to earlier observations conducted in drier heat that fluid ingestion attenuates hyperthermia during exercise in a heat stressful condition (Montain and Coyle, 1992; Below et al., 1995; Armstrong et al., 1997; Sawka et al., 2012).

The findings of the present study provide new insight in better understanding the efficacy of fluid ingestion on thermoregulatory responses in two different heat stress conditions (i.e., warm-dry and warm-humid). Numerous earlier guidelines on fluid ingestion (Casa et al., 2000; Sawka et al., 2007, 2015) do not specifically comment on the efficacy of fluid ingestion in attenuating heat strain under different thermal profiles. The present study demonstrated that in the humid condition with a reduced $\mathrm{E}_{\max }$, there is an increase in the amount of sweat dripping off the skin instead of 
being evaporated (Candas et al., 1979; Frye and Kamon, 1983; Alber-Wallerström and Holmér, 1985). Therefore, the notion that fluid ingestion during exercise heat stress will attenuate hyperthermia by enhancing sweat evaporation does not take place in a humid condition. Instead, the physical characteristics of the environment would dictate the efficacy of fluid ingestion in attenuating hyperthermia. This was evident in the current study where a higher WBSR recorded in the humid condition did not result in a lowering of skin temperature that would have increased the gradient between the core and the skin, thus promoting heat transfer and lowering body heat storage.

Instead, skin temperature remained elevated in the humid condition due to the lower $\mathrm{E}_{\max }$. The current observation that fluid ingestion was not effective in attenuating thermal strain and improving endurance performance was consistent with several earlier studies. Kay and Marino (2003) reported that water ingestion failed to improve self-paced exercise performance in a hot-humid environment. In addition, several other field-based studies conducted in hot-humid environments had reported no association between fluid intake and percent dehydration with core temperature response (Byrne et al., 2006; Lee et al., 2010). This further supports numerous reports that have proposed that a certain amount of dehydration is tolerable during running exercise where thermoregulatory function is not significantly impaired until a critical level of dehydration has accrued. Davies and Thompson (1986) recorded a 5.5\% body weight deficit at the end of $4 \mathrm{~h}$ strenuous running exercise with core temperature at no point exceeding $39.3^{\circ} \mathrm{C}$. Based on this evidence it is suggested that the level of dehydration may have a relatively small influence on core temperature during exercise if the subjects are euhydrated at the onset of exercise. Several more recent findings have consistently demonstrated that a body mass loss of up to $4 \%$ has been well tolerated during prolonged exercise and did not appear to have any detrimental effect on exercise performance (Zouhal et al., 2011; Del Coso et al., 2014; Hoffman and Stuempfle, 2014).

Guidelines on fluid ingestion during exercise have been formulated mainly based on the notion that dehydration impairs endurance exercise performance (Casa et al., 2000; Sawka et al., 2007, 2015) as dehydration level exceeds $2 \%$ of body mass (Montain and Coyle, 1992; Below et al., 1995; Ebert et al., 2007; Merry et al., 2010; Sawka et al., 2012; Cheuvront and Kenefick, 2014). On the contrary, several other researchers have consistently shown that in well-trained cyclists who were euhydrated at the onset of exercise and went on to cycle for up to $60 \mathrm{~min}$, their cycling performance was not compromised within an ambient temperature of up to $33^{\circ} \mathrm{C}$ and $60 \% \mathrm{RH}$ despite their dehydration level reaching 4\% (Noakes, 2007; Goulet, 2011, 2013; Wall et al., 2013).

The level of plasma volume in this study was well maintained during exercise despite the difference in dehydration rate between the NF and FI trial. This observation is consistent with several earlier studies that have shown plasma volume can be partially defended at an even larger percentage of body weight deficit during intense $\left(65-75 \% \dot{\mathrm{VO}}_{2} \max \right)$ running exercise (Costill et al., 1970; Sawka et al., 1980; Kolka et al., 1982;
Gass et al., 1983; Davies and Thompson, 1986) The magnitude of dehydration in the present study was much smaller than these earlier studies that have reported a stable plasma volume. For instance, Costill et al. (1970), Sawka et al. (1980), and Kolka et al. (1982) recorded a stable plasma volume during exercise at an even higher percent body weight deficit of $4 \%, 4.8-6.8 \%$ and $7 \%$, respectively. The ability to defend the level of plasma volume in light of increasing dehydration level has been associated with the participants euhydration level at the onset of exercise as well as the ability to shift body water interstitially. In addition, the release of water from glycogenolysis, metabolic water production and the redistribution of water from inactive skeletal muscle has also been reported to assist in maintaining plasma volume during exercise (Pivarnik et al., 1984).

\section{CONCLUSION}

In conclusion, the current study has demonstrated that the efficacy of fluid ingestion in attenuating thermoregulatory and circulatory stress during prolonged exercise is potentially dependent on the physical characteristics of the environment. Runners who started exercise in a euhydrated state did not gain any ergogenic benefit for the $1 \mathrm{~h}$ of exercise from fluid ingestion. Runners in our study who had experienced dehydration ranging from $1.8 \%$ to $2.0 \%$ did not improve their performance. This was in contrast with the wellestablished notion that dehydration of up to $2 \%$ of body weight loss will increase thermoregulatory and circulatory stress leading to impairment in endurance exercise performance (Casa et al., 2000; Sawka et al., 2007, 2015). Instead, our finding was in agreement with earlier reports of Kay and Marino (2003) as well as Lee et al. (2010) which failed to observe any ergogenic benefit of fluid ingestion on exercise performance in humid heat.

This study highlights the need to consider the characteristics of the environment in evaluating the efficacy of fluid ingestion in attenuating thermoregulatory and circulatory strain during exercise and its implications on exercise performance. Future recommendations relating to fluid ingestion during exercise should now discuss the impact of environmental conditions.

\section{ETHICS STATEMENT}

The University of Sydney Human Ethics Committee approved the experimental protocol for this study (Ref. No: 99/05/46), which conformed to the current Declaration of Helsinki guidelines.

\section{AUTHOR CONTRIBUTIONS}

ACM, SS, and MT involved in the design and implementation of the study. TM and HY assisted the corresponding author in the data analysis. All authors contributed in preparing the manuscript with HY preparing the figures. 


\section{REFERENCES}

Alber-Wallerström, B., and Holmér, I. (1985). Efficiency of sweat evaporation in unacclimatized man working in a hot humid environment. Eur. J. Appl. Physiol. 54, 480-487. doi: 10.1007/bf00422956

Armstrong, L. E., Maresh, C. M., Gabaree, C. V., Hofman, J. R., Kavouras, S. A., Kenefick, R. W., et al. (1997). Thermal and circulatory responses during exercise: effects of hypohydration, dehydration, and water intake. J. Appl. Physiol. 82, 2028-2035. doi: 10.1152/jappl.1997.82.6.2028

Atkins, K., and Thompson, M. W. (2000). A spreadsheet for partitional calorimetry. Sportscience 4. Available at: sportsci.org/jour/0003/ka.html

Below, P.R., Mora-Rodriguez, R., Gonzalez-Alanso, J., and Coyle, E.F. (1995). Fluid and carbohydrate ingestion independently improve performance during 1 hour of intense exercise. Med. Sci. Sports Exerc. 27, 200-210.

Borg, G.A. (1982). Psychophysical bases of perceived exertion. Med. Sci. Sports Exerc. 14, 377-381.

Byrne, C., Lee, J.K., Chew, S.A., Lim, C.L., and Tan E.Y. (2006). Continuous thermoregulatory responses to mass participation distance running in heat. Med. Sci. Sports Exerc. 38, 803-810. doi: 10.1249/01.mss.0000218134.74238.6a

Candas, V., Libert, J. P., and Vogt, J. J. (1979). Human skin wettedness and evaporative efficiency of sweating. J. Appl. Physiol. 46, 522-528. doi: 10.1152/ jappl.1979.46.3.522

Casa, D. J., Armstrong, L. E., Hillman, S. K., Montain, S. J., Reiff, R. V., Rich, B. S., et al. (2000). National athletic trainers' association position statement: fluid replacement for athletes. J. Athl. Train. 35, 212-224.

Che Muhamed, A. M, Atkins, K, Stannard, S. R., Mündel, T., and Thompson, M. W. (2016). The effects of a systematic increase in relative humidity on thermoregulatory and circulatory responses during prolonged running exercise in the heat. Temperature 18, 455-464. doi: 10.1080/23328940.2016.1182669

Cheuvront, S. N., and Kenefick, R. W. (2014). Dehydration: physiology, assessment and performance effects. Compr. Physiol. 4, 257-285. doi: 10.1002/cphy. c130017

Colin, J., Timbal, J., Houdas, Y., Boutelier, C., and Guieu, J. D. (1971). Computation of mean body temperature from rectal and skin temperature. J. Appl. Physiol. 31, 484-489. doi: 10.1152/jappl.1971.31.3.484

Collier, C. R. (1956). Determination of mixed venous CO2 tensions by rebreathing. J. Appl. Physiol. 9, 25-29. doi: 10.1152/jappl.1956.9.1.25

Costill, D. L., Kammer, W. F., and Fisher, A. (1970). Fluid ingestion during distance running. Arch. Environ. Health 21, 520-525. doi: 10.1080/00039896.1970. 10667282

Davies, C. T. (1979). Influence of skin temperature on sweating and aerobic performance during severe work. J. Appl. Physiol. 47, 770-777. doi: 10.1152/ jappl.1979.47.4.770

Davies, C. T, and Thompson, M. W. (1986). Physiological responses to prolonged exercise in ultramarathon athletes. J. Appl. Physiol. 61, 611-617. doi: 10.1152/ jappl.1986.61.2.611

Del Coso, J., Gonzalez, C., Abian-Vicen, J., Salinero Martin, J. J., Soriano, L., Areces, F., et al. (2014). Relationship between physiological parameters and performance during a half-ironman triathlon in the heat. J. Sports Sci. 32, 1680-1687. doi: 10.1080/02640414.2014.915425

Dill, D. B., and Costill, D. L. (1974). Calculation of percentage changes in volumes of blood, plasma and red cells in dehydration. J. Appl. Physiol. 37, 247-248. doi: 10.1152/jappl.1974.37.2.247

DuBois, D. and DuBois, E.F. (1916). A formula to estimate the approximate surface area if height and weight be known. Arch. Int. Med. 17, $863-871$.

Ebert, T.R., Martin, D.T., Bullock, N., Mujika, I., Quod, M.J., Farthing, L.A., Burke, L.M. et al. (2007) Influence of hydration status on thermoregulation and cycling hill climbing. Med. Sci. Sports Exerc. 39, 323-329. doi: 10.1249/01.mss. 0000247000.86847.de

Fanger, P. O. (1970). Thermal Comfort. New York, NY: McGraw-Hill.

Frye, A. J., and Kamon, E. (1983). Sweating efficiency in acclimated men and women exercising in humid and dry heat. J. Appl. Physiol. 54, 972-977. doi: 10.1152/jappl.1983.54.4.972

Gass, G. C., Camp, E. M., Watson, J., Eager, D., Wicks, L., and Ng, A. (1983). Prolonged exercise in highly trained female endurance runners. Int. J. Sports Med. 4, 241-246. doi: 10.1055/s-2008-1026042
Goulet, E.D.B. (2011). Effect of exercise-induced dehydration on time-trial exercise performance: a meta-analysis. Br. J. Sports Med. 45, 1149-1156. doi: 10.1136/ bjsm.2010.077966

Goulet, E.D.B. (2013). Effect of exercise-induced dehydration on endurance performance: evaluating the impact of exercise protocols on outcomes using a meta-analytic procedure. Br. J. Sports Med. 47, 679-686. doi: 10.1136/bjsports2012-090958

Hoffman, M.D. and Stuempfle, K.J. (2014). Hydration strategies, weight change and performance in a $161 \mathrm{~km}$ ultramarathon. Res. Sports Med. 22, 213-225. doi: 10.1080/15438627.2014.915838

Kay, D., and Marino, F. E. (2003). Failure of fluid ingestion to improve self-paced exercise performance in moderate to warm humid environments. J. Therm. Biol. 28, 29-34. doi: 10.1016/s0306-4565(02) 00032-3

Kolka, M. A., Stephenson, L. A., and Wilkerson, J. E. (1982). Erythrocyte indices during a competitive marathon. J. Appl. Physiol. 52, 168-172. doi: 10.1152/ jappl.1982.52.1.168

Lee, J. K., Nio, A. Q., Lim, C. L., Teo, E. Y., and Byrne, C. (2010). Thermoregulation, pacing and fluid balance during mass participation distance running in a warm and humid environment. Eur. J. Appl. Physiol. 109, 887-898. doi: 10.1007/ s00421-010-1405-y

Maughan, R. J., Otani, H., and Watson, P. (2012). Influence of relative humidity on prolonged exercise capacity in a warm environment. Eur. J. Appl. Physiol. 112, 2313-2321. doi: 10.1007/s00421-011-2206-7

McIntyre, D. A. (1980). Indoor Climate. London: Applied Science Publishers.

Merry, T. L., Ainslie, P. N., and Cotter, J. D. (2010). Effects of aerobic fitness on hypohydration-induced physiological strain and exercise impairment. Acta Physiol. 198, 179-190. doi: 10.1111/j.1748-1716.2009. 02051.x

Mitchell, J. W., Nadel, E. R., and Stolwijk, J. A. (1972). Respiratory weight losses during exercise. J. Appl. Physiol. 32, 474-476. doi: 10.1152/jappl.1972.32. 4.474

Montain, S. J., and Coyle, E. F. (1992). Influence of graded dehydration on hyperthermia and cardiovascular drift during exercise. J. Appl. Physiol. 73, 1340-1350. doi: 10.1152/jappl.1992.73.4.1340

Moyen, N.E., Ellis, C.L., Ciccone, A.B., Thurston, T.S., Cochrane, K.C., Brown, L.E., Coburn, J.W. et al. (2014). Increasing relative humidity impacts lowintensity exercise in the heat. Aviat. Space Environ. Med. 85, 112-119. doi: 10.3357/asem.3787.2014

Noakes, T. D. (2007). Drinking guidelines for exercise: what evidence is there that athletes should drink "as much as tolerable", "to replace the weight lost during exercise" or "ad libitum"? J. Sports Sci. 25, 781-796. doi: 10.1080/ 02640410600875036

Pivarnik, J. M., Leeds, E. M., and Wilkerson, J. E. (1984). Effects of endurance exercise on metabolic water production and plasma volume. J. Appl. Physiol. 56, 613-618. doi: 10.1152/jappl.1984.56.3.613

Ramanathan, N. L. (1964). A new weighting system for mean surface temperature of the human body. J. Appl. Physiol. 19, 531-533. doi: 10.1152/jappl.1964.19. 3.531

Sawka, M. N., Cheuvront, S. N., and Kenefick, R. W. (2012). High skin temperature and hypohydration impair aerobic performance. Exp. Physiol. 97, 327-332. doi: 10.1113/expphysiol.2011.061002

Sawka, M.N., Burke, L.M., Eichner, E.R., Maughan, R.J., Montain, S.J. and Stachenfeld, N.S. (2007). American college of sports medicine position stand: exercise and fluid replacement. Med. Sci. Sports Exerc. 39, 377-390. doi: 10. 1249/mss.0b013e31802ca597

Sawka, M.N., Cheuvront, S.N. and Kenefick, R.W. (2015). Hypohydration and human performance: impact on environment and physiological mechanisms. Sports Med. 45 (Suppl. 1), S51-S60. doi: 10.1007/s40279-0150395-7

Sawka, M.N., Knowlton, R.G. and Glaser, R.M. (1980). Body temperature, respiration, and acid-base equilibrium during prolonged running. Med. Sci. Sports Exerc. 12, 370-374. doi: 10.1249/00005768-19802500000013

Sawka, M.N., and Pandolf, K.B. (1990). "Effects of body water loss on exercise performance and physiological functions," in Fluid Homeostasis During Exercise eds C.V. Gisolfi and D.R. Lamb (Carmel: Benchmark Press). 
Siri, W.E. (1961). "Body composition from fluid space and density," in Techniques for Measuring Body Composition eds J. Brozek and A. Hanschel (Washington, DC: National Academy of Science), 223-244.

Wall, B.A., Watson, G., Peiffer, J.J., Abbiss, C.R., Siegel, R. and Laursen, P.B. (2013). Current hydration guidelines are erroneous: dehydration does not impair exercise performance in the heat. Br. J. Sports Med. 49, 1077-1083. doi: 10.1136/bjsports-2013092417

Zouhal, H., Groussard, C., Minter, G., Vincent, S., Cretual, A., GratasDelamarche, A., Delamarche, P. et al. (2011). Inverse relationship between percentage body weight change and finising time in 643 forty-two-kilometre marathon runners. Br. J. Sports Med. 45, 1101-1105. doi: 10.1136/bjsm.2010. 074641
Conflict of Interest Statement: The authors declare that the research was conducted in the absence of any commercial or financial relationships that could be construed as a potential conflict of interest.

The reviewer ZS declared a past co-authorship with one of the authors TM to the handling Editor.

Copyright (c) 2019 Che Muhamed, Yusof, Stannard, Mündel and Thompson. This is an open-access article distributed under the terms of the Creative Commons Attribution License (CC BY). The use, distribution or reproduction in other forums is permitted, provided the original author(s) and the copyright owner(s) are credited and that the original publication in this journal is cited, in accordance with accepted academic practice. No use, distribution or reproduction is permitted which does not comply with these terms. 